\title{
Evidências de Validade da Versão Reduzida do Coping Orientation to Problems Experienced Inventory (COPE) com Trabalhadores Brasileiros
}

\author{
Evidencias de validez de la versión reducida del Coping \\ Orientation to Problems Experienced Inventory (COPE) con \\ trabajadores brasileños
}
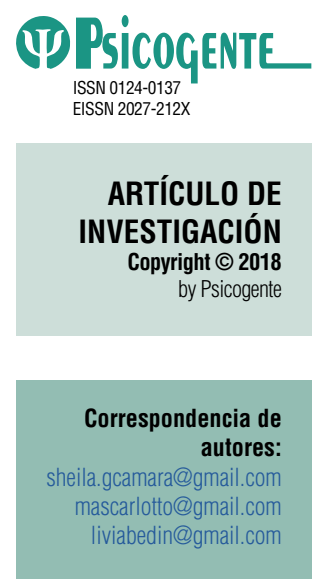

Recibido: $10-07-17$ Aceptado: 23-03-18 Publicado: 08-01-19

\section{Validity evidences of the Coping Orientation to Problems Experienced Inventory (COPE) reduced version with Brazilian workers}

Sheila Gonçalves Câmara (iP)

Universidade Federal de Ciências da Saúde de Porto Alegre, Porto Alegre, Brasil

Mary Sandra Carlotto iD

Universidade do Vale do Rio dos Sinos, São Leopoldo, Brasil

Lívia Maria Bedin (iD

Universidade Federal do Rio Grande do Sul, Porto Alegre, Brasil

Resumo

Objetivo: Coping é definido como os esforços cognitivos e comportamentais para controlar, vencer, tolerar ou reduzir demandas internas e externas que excedem as capacidades da pessoa em termos de estresse. Este artigo propõe uma versão reduzida do Inventário COPE.

Método: Participaram deste estudo transversal 524 trabalhadores de instituições localizadas em Porto Alegre e Região Metropolitana (RS), Brasil, selecionados por acessibilidade. Destes, 57,6\% eram mulheres com idades entre 16 e 67 anos ( $M=30,7 ; D P=10)$. Como instrumentos foram utilizados o Inventario COPE e um questionário de dados sociodemográficos e laborais. A validade de conteúdo foi analisada através do método de juízes. A validade de construto foi analisada mediante análise fatorial confirmatória e confiabilidade.

Resultados: Em termos da validade de conteúdo, os juízes confirmaram a abrangência teórica e conceitual da escala, considerando os elementos apropriados para avaliar coping. A análise fatorial confirmatória demonstrou índices de ajustes adequados para o modelo de três dimensões: RMSEA =0,05 (I.C = $0,04-0,06) ; C F I=0,94$ e alfas satisfatórios para o modelo composto por 18 itens, seis que avaliam estratégias de coping evitativo $(\alpha=0,71)$, seis que avaliam as estratégias com foco na emoção $(\alpha=0,78)$ e seis, estratégias com foco no problema $(\alpha=0,81)$. Tais resultados indicam que a COPE possui propriedades psicométricas adequadas para o estudo do coping no Brasil. Conclusões: $\mathrm{O}$ uso da versão reduzida do COPE pode contribuir para uma avaliação mais ágil e efetiva no que tange às estratégias de coping na realidade brasileira.

Palavras-chave: Coping, Escala, Adaptação de instrumentos, Análise fatorial.

Cómo citar este artículo (APA):

Gonçalves Câmara, S., Carlotto, M. S. y Bedin, L. M. (2019). Evidências de Validade da Versão Reduzida do Coping Orientation to Problems Experienced

Inventory (COPE) com Trabalhadores Brasileiros. Psicogente 22(41), 1-18. https://doi.org/10.17081/psico.22.41.3301 
Resumen

Objetivo: Afrontamiento se define como los esfuerzos cognitivos y conductuales para controlar, vencer, tolerar o reducir demandas internas y externas que exceden las capacidades de la persona en términos de estrés. Este artículo de investigación tuvo como objetivo analizar las evidencias de validez de la versión reducida de 18 ítems del inventario COPE.

Método: Participaron de ese estudio transversal 524 trabajadores de instituciones localizadas en Porto Alegre y Región Metropolitana (RS), Brasil, seleccionados por accesibilidad. De esos, 57,6\% eran mujeres con edades entre 16 y 67 años $(M=30,7 ; D P=10)$. Como instrumentos fueron utilizados el Inventario COPE y un cuestionario de datos sociodemográficos y laborales. Se analizó la validez de contenido a través del método de jueces. La validez de constructo se realizo mediante el análisis factorial confirmatorio y la confiabilidad.

Resultados: En términos de validez de contenido, los jueces confirmaron el ámbito teórico y conceptual de la escala, considerando los elementos apropiados para evaluar coping. El análisis factorial confirmatorio demostró índices de ajuste adecuados para el modelo de tres dimensiones: RMSEA =0,05 (I.C. = 0,04 $0,06) ; C F I=0,94$, así como alfas satisfactorios para el modelo compuesto por 18 ítems, seis que evalúan estrategias de afrontamiento de evitación $(\alpha=0,71)$, seis que evalúan las estrategias con foco en la emoción $(\alpha=0,78)$ y seis estrategias con foco en el problema $(\alpha=0,81)$. Los resultados indican que la COPE tiene propiedades psicométricas adecuadas para el estudio del afrontamiento en Brasil.

Conclusiones: La utilización de la versión reducida del COPE puede contribuir para una evaluación más ágil y efectiva en lo que concierne a las estrategias de afrontamiento en la realidad brasileña.

Palabras clave: Afrontamiento, Escala, Adaptación de instrumentos, Análisis factorial.

Abstract

Objective: Coping is defined as cognitive and behavioral efforts to control, win, tolerate or reduce internal or external demands that exceed person's resources in relation with stress. This paper proposes COPE Inventory as a reduced version including 18 items.

Method: A cross-sectional study was conducted; 524 workers from institutions located in Porto Alegre and Metropolitan Region (MR), Brazil, selected by accessibility were sampled. $57.6 \%$ of the participants, were women between $16-67$ years old $(M=30.7, S D=10)$. As instruments, the COPE Inventory and a sociodemographic and labor data questionnaire were used. Content validity was analyzed through the judges method. The construct validity was analyzed by confirmatory factor analysis.

Results: In terms of content validity, the judges confirmed the theoretical and conceptual scope of the scale, considering the elements appropriate to evaluate coping. The confirmatory factor analysis showed adequate adjustment indices for the three-dimensional model: $R M S E A=0.05$ (C. I. $=0.04-0.06$ ); $C F I=0.94$ and satisfactory alphas to the model composed by 18 items, six that evaluate evitative coping strategies $(\alpha=0.71)$, six that evaluate emotion focused coping $(\alpha=0.78)$ and six evaluating problem focused coping $(\alpha=0.81)$ were identified. These results show that COPE has adequate psychometric properties for coping study in Brazil.

Conclusions: COPE in a reduced version use may contribute to a more agile and more effective evaluation related to the use of coping strategies in Brazilian reality.

Keywords: Coping, Scale, Instrument's adaptation, Factorial analysis.

\section{INTRODUÇÃO}

A maneira pela qual as pessoas lidam com situações estressantes em suas vidas representa um tema cada vez mais relevante na atualidade, uma vez que sua estreita relação com o bem-estar psicológico tem sido demontrada (O’Driscoll, 2013; Sinha, Lacadie, Constable, \& Seo, 2016). As estratégias de enfrentamento do estresse, ou coping, desempenham um importante papel na relação entre o estresse e o processo de saúde-doença (Carver \& Scheier, 
2017; Lazarus \& Folkman, 1984; Marroquín, Tennen, \& Stanton, 2017; Murta \& Tróccoli, 2007; Roche \& Ogden, 2017).

Coping tem sido definido como os esforços cognitivos e comportamentais para controlar, vencer, tolerar ou reduzir as demandas internas ou externas específicas que são avaliadas como excedendo os recursos da pessoa (Lazarus \& Folkman, 1984). São esforços para prevenir ou diminuir a ameaça, dano e perda, ou para reduzir o sofrimento associado a situações de estresse (Carver \& Connor-Smith, 2010). Consiste, dessa forma, em um construto aplicado importante na relação dos indivíduos com seus contextos sociais e culturais (Frydenberg, 2017).

De acordo com o modelo tradicional, proposto por Folkman e Lazarus (1980), as estratégias de enfrentamento podem ser classificadas em dois tipos, conforme sua função: focadas na emoção ou no problema. O coping focalizado na emoção é um esforço para regular o estado emocional que é associado ao estresse, ou é o resultado de eventos estressantes. Este esforço tem por objetivo alterar o estado emocional da pessoa na tentativa de reduzir a sensação física desagradável do estado de estresse (Diniz \& Zanini, 2010; Noda, Takahashi, \& Murai, 2018; Reeck, Ames, \& Ochsner, 2016; Veloso-Besio, Caqueo-Arancibia, Caqueo-Urízar, Muñoz-Sánchez, \& VillegasAbarzúa, 2010). O coping focalizado no problema é um esforço para mudar a situação que deu origem ao estresse e tem como função alterar o problema existente na relação entre a pessoa e o ambiente, o qual está causando a tensão (Folkman \& Lazarus, 1980). A ação é dirigida ao próprio estressor visando removê-lo ou diminuir seu impacto (Carver \& Connor-Smith, 2010).

Ambas as estratégias, com foco no problema e na emoção, podem auxiliar-se mutuamente. A utilização do coping focado no problema tem impacto emocional ao diminuir a sensação de ameaça e a angústia que se deriva desta. Já o enfrentamento mediante estratégias focalizadas na emoção, por sua capacidade de diminuir as sensações negativas, possibilita a consideração da situação estressora de maneira mais objetiva e, portanto, possibilita estratégias com foco no problema (Juth, Dickerson, Zoccola, \& Lam, 2015). Embora exista uma tendência das pessoas em utilizar preferencialmente uma das formas, o uso de ambas é recorrente, demonstrando que as duas formas de enfrentamento não são totalmente independentes (Biggs, Brough, \& Drummond, 2017; Lazarus, 2006). 
Outra classificação sobre coping refere-se à aproximação ou evitação frente à situação estressora ou emoções associadas a ela. As estratégias de aproximação englobam basicamente estratégias com foco no problema, além de algumas com foco na emoção que se referem a estratégias de busca de melhores condições pessoais para fazer frente ao problema. Já as estratégias evitativas podem ser compreendidas como unicamente com foco na emoção, pelas quais a pessoa afasta o estressor, de maneira a não ter que lidar com ele (Carver \& Connor-Smith, 2010).

De acordo com Endler e Parker (1990), coping pode ser classificado em três tipos: orientado à tarefa (foco na resolução do problema), orientado à emoção (foco nas emoções negativas derivadas da situação), e orientado à evitação (foco na tentativa de evitar o problema). Em termos adaptativos, as estratégias evitativas, embora apresentem funcionalidade em curto prazo, em médio e longo prazo, podem produzir efeitos negativos (Schaufeli \& Enzmann, 1998).

No modelo de avaliação de estratégias de coping proposto por Carver et al. (1989), há uma certa fluidez no que se refere à sobreposição das categorias de foco no problema e na emoção e aproximação e evitação, na tentativa de contemplar a todas conjuntamente. Os autores propuseram um instrumento multidimensional de avaliação de respostas a situações estressantes, tendo por base os modelos de autorregulação de comportamento (Carver \& Scheier, 1981) e de estresse e coping (Lazarus \& Folkman, 1984). Sua concepção parte do princípio de que as estratégias de enfrentamento ao estresse são relativamente estáveis em relação a diferentes tipos de estressores.

O Coping Orientation to Problems Experienced Inventory - COPE (Carver et al., 1989), originalmente, foi composto por 52 itens e 13 escalas subdivididas em três dimensões: foco no problema ( 5 escalas), foco na emoção (5 escalas) e estratégias de pouca utilidade (três escalas). As escalas de enfrentamento com foco no problema são: enfrentamento ativo, planejamento, supressão de atividades concorrentes, contenção de enfrentamento e busca de apoio social instrumental. As escalas de enfrentamento com foco na emoção são: busca de apoio social emocional, reinterpretação positiva, aceitação, negação e voltar-se para a religião. As escalas consideradas de pouca utilidade são: ventilação de emoções, desligamento comportamental e desligamento mental. 
No desenvolvimento do instrumento, um item relativo ao uso de álcool e drogas foi incluído como potencialmente pertencente à escala de desligamento mental, mas este não saturou em seu fator de origem e foi apresentado separadamente no estudo original (Carver et al., 1989). No entanto, os autores referem que diversas estratégias foram contempladas no processo de concepção da COPE e, na versão completa do inventário, disponível na página do professor Charles S. Carver, na Universidade de Miami (http://www.psy.miami.edu/faculty/ccarver/sclCOPEF.html), há uma escala específica para uso de substâncias, composta por 4 itens. Além dessa escala, o instrumento atual conta também com uma escala denominada de humor.

O inventário apresenta a flexibilidade de avaliar estratégias de enfrentamento de forma disposicional ou situacional, dependendo da forma como é aplicado. Na forma disposicional os participantes são solicitados a respondê-lo de acordo com a forma como habitualmente reagem frente às situações propostas. Na forma situacional, os participantes respondem às mesmas situações de acordo com a maneira como reagiram recentemente. A diferença entre as duas formas está nas instruções do inventário e na forma verbal como este é apresentado. Nos estudos de construção e de evidências de validade, na forma disposicional, todas as escalas apresentaram coeficientes de consistência interna superiores a 0,60 , sendo estes ainda mais elevados na forma situacional (Carver et al., 1989).

Em revisão sistemática sobre a frequência de uso de escalas de coping em artigos publicados em revistas científicas entre os anos de 1998 e 2010, Kato (2013) identificou que o COPE foi o mais utilizado. Em 75,5\% dos 404 artigos que utilizaram o inventário, em sua versão integral ou abreviada, a estrutura da escala foi modificada em relação ao proposto por Carver et al. (1989) ou Carver (1997). Os estudos apresentaram novas escalas, formadas a partir de análise fatorial ou utilizaram apenas algumas das subescalas do inventário. As estruturas fatoriais encontradas variam tanto em termos de quantidade de fatores quanto de conteúdo (Cabral \& Matos, 2010; Greer, 2007; Kallasmaa \& Pulver, 2000; Litman, 2006; Lyne \& Roger, 2000). Modelos de três fatores tem sido identificados em estudos como os de Lyne e Roger (2000), que os denominou de coping racional, coping emocional e coping evitativo. Kallasmaa e Pulver (2000) também identificaram uma estrutura trifatorial e denominaram os fatores de coping com foco na tarefa, coping de evitação e coping social/emocional. Dobreva-Martinova, Villenueve, 
Strickland e Matheson (2002) utilizaram o COPE com três fatores: coping com foco no problema, coping com foco na emoção e coping evitativo.

Dadas as dificuldades de inserção da COPE completa em baterias com mais instrumentos, devido ao seu tamanho, Carver (1997) desenvolveu uma versão reduzida, a Brief COPE. Essa foi desenvolvida para facilitar a administração, com redução de tempo dispendido (Baumstarck, et al., 2017). Nessa versão houve a exclusão de duas escalas da COPE completa e o acréscimo de uma nova escala, além da supressão de dois itens em cada escala. As escalas de contenção de enfrentamento e supressão de atividades concorrentes foram retiradas, pois, de acordo com a avaliação do autor, apresentaram pouca relevância em estudos anteriores e tinham redundância em relação à escala de enfrentamento ativo. Carver também elaborou dois itens referentes a estratégias de auto-culpar-se, criando uma nova escala. Para tanto, ele se embasou na literatura, que aponta que as tendências de auto-culpa são preditoras de desequilíbrio frente a situações de estresse. Para a seleção dos itens em cada uma das demais escalas, o autor considerou, primeiramente, a carga fatorial dos itens em seus fatores na análise fatorial do estudo original (Carver, et al., 1989). Como um critério adicional, ele utilizou sua experiência de campo com o instrumento completo, considerando a compreensibilidade dos itens entre populações de não estudantes. Assim, a escala ficou composta por 28 itens divididos em 14 escalas. Em termos de consistência interna, todas as escalas alcançaram alfas superiores a 0,50.

Dentre as escalas de enfrentamento disponíveis, existem algumas amplamente aplicáveis, como o COPE (Carver, et al., 1989) e a Estratégias de coping (WCQ) (Folkman \& Lazarus, 1985). Diversas escalas para avaliar estratégias de enfrentamento são utilizadas nas investigações sobre estresse em áreas como a medicina, enfermagem e psicologia, contribuindo para o desenvolvimento do conhecimento sobre a relação entre estresse e saúde (Kato, 2013). De acordo com o autor, é por esse motivo que muitos pesquisadores têm avaliado as propriedades psicométricas de escalas de enfrentamento na tentativa de aperfeiçoá-las.

No Brasil, estudos têm sido realizados com o Inventário de Estratégias de Coping, de Lazarus e Folkman (1984), a Escala de Coping Ocupacional, de Latack (1986), adaptada para o Brasil por Pinheiro, Tróccoli e Tamayo (2003), a Escala Modos de Enfrentamento de Problemas (EMEP), de Seidl, Tróccoli e Zannon (2001) e pelo Inventário COPE, de Carver et al. (1989). Outros 
instrumentos têm sido desenvolvidos ou validados especificamente para a população adolescente, como é o caso do Inventário Multifatorial de Coping para Adolescentes-IMCA-43 (Antoniazzi, 2000) e a Escala Brasileira de Coping para Adolescentes (Câmara \& Carlotto, 2016).

De maneira geral, as escalas de avaliação de estratégias de enfrentamento disponíveis no Brasil apresentam especificidades no que se refere ao modelo de Coping adotado, de maneira que, em alguns casos, a definição das estratégias avaliadas não permite uma classificação geral mais ampla, em termos de foco no problema e na emoção, por exemplo (Melo, Carlotto, Rodríguez, \& Diehl, 2016). Outros instrumentos estão diretamente vinculados ao contexto específico para o qual foram desenvolvidos, como o instrumento diretamente vinculado ao âmbito ocupacional ou os instrumentos direcionados a adolescentes. Além dessas questões, existem as de ordem prática, referentes à sua compreensão pela população em estudo e sua extensão (Morero, Bragagnollo \& Santos, 2018).

O inventário COPE, nesse sentido, apresenta a vantagem de ser uma medida de avaliação geral com diversas escalas que podem ser agrupadas em três fatores de segunda ordem ou combinados de acordo com as especificidades de cada pesquisa e da população em estudo. Além disso, seus índices de confiabilidade, no estudo original (Carver et al., 1989) e em outros que utilizaram o instrumento (Dobreva-Martinova et al., 2002; Lyne \& Roger, 2000), são satisfatórios. A principal desvantagem desse instrumento é sua extensão (Carver, 1997). Também é importante considerar que há um elevado número de escalas com poucos itens ( 4 por escala) para avaliar as três dimensões principais (foco no problema -5 escalas, foco na emoção - 5 escalas e evitação -5 escalas).

A Brief COPE foi a alternativa proposta por Carver (1997) para resolver o problema de extensão da COPE completa. Essa mantém uma ampla gama de estratégias de enfrentamento. No entanto, a versão foi elaborada utilizando uma amostra reduzida de pessoas e a estratégia de manter um grande número de escalas (14), com apenas dois itens cada uma, pode ser um aspecto que incida na confiabilidade do instrumento em sua utilização com outras amostras. Estudo que propôs uma versão francesa da Brief COPE identificou uma estrutura de quatro fatores: suporte social, solução de problemas, evitação e pensamento positivo, com coeficientes alfa de Cronbach que 
variaram de 0,64 a 0,82. No entanto, a amostra foi composta por pessoas que estavam enfrentando um momento de crise vital (Baumstarck et al., 2017).

De acordo com o exposto, o presente estudo, observacional e transversal, teve por objetivo propor uma versão reduzida do Inventário COPE completo, considerando o modelo de três dimensões de estratégias de enfrentamento (foco no problema, foco na emoção e evitação), de acordo com a categorização utilizada pelos autores do instrumento (Carver et al., 1989) e outros pesquisadores (Dobreva-Martinova et al., 2002; Lyne \& Roger, 2000). Com isso, pretende-se propor uma versão reduzida do instrumento, reduzindo o tempo de coleta, porém mantendo os pressupostos teóricos do construto de coping, de acordo com o modelo adotado. Utilizou-se uma amostra de trabalhadores do sul do Brasil por considerar-se que essa é uma população bastante heterogênea, que contempla os critérios para o estudo.

\section{MÉTODO}

\subsection{Participantes}

A amostra por acessibilidade foi composta por 524 trabalhadores de instituições localizadas em Porto Alegre e Região Metropolitana (RS), Brasil, contatados via e-mail e redes sociais. Como critério de inclusão, os trabaIhadores deveriam estar em exercício profissional há mais de seis meses. A maioria foi composta de mulheres $(57,6 \%)$, sem companheiro(a) $(63,6 \%)$, que não possuíam filhos (60,5\%), com escolaridade em nível superior $(67,1 \%)$. A idade dos participantes variou de 16 a 67 anos ( $M=30,7 ; D P=10)$. Em média, trabalhavam na profissão há 7,6 anos ( $D P=6,3$ ) com uma variação de um a 35 anos. A maioria exercia funções administrativas $(57,1 \%)$ e trabalhava em empresas de médio a grande porte $(80,4 \%)$.

\subsection{Instrumentos}

\subsubsection{Inventário COPE (Carver et al., 1989)}

Tem como objetivo identificar as estratégias de enfrentamento utilizadas para lidar com estressores. A escala original encontra-se disponível no site do Department of Psychology College of Arts and Ciences da Universidade de Miami (http://www.psy.miami.edu/faculty/ccarver/sclCOPEF.html). O inventário totaliza 60 itens com sistema de pontuação de 1 (não costumo fazer isso nunca) a 4 (costumo fazer isso muito). O inventário se constitui de 15 fatores (quatro itens para cada fator) que representam estratégias de enfrenta- 
mento: coping ativo, planejamento, supressão de atividades concomitantes, coping moderado, busca de suporte social por razões instrumentais, busca de suporte social por razões emocionais, reinterpretação positiva e crescimento, aceitação, retorno para a religiosidade, foco na expressão das emoções, negação, comportamento descomprometido, desengajamento mental, humor e uso de substâncias. Na escala original, a fidedignidade encontrada em relação à consistência interna variou de 0,62 a 0,92 nos 15 fatores, o que foi considerado satisfatório pelos autores (Carver et al., 1989).

\subsubsection{Questionário de dados sociodemográficos e laborais}

Foi elaborado, especificamente para o estudo, um inquérito de dados sociodemográficos que constava das seguintes variáveis: sexo, idade, situação conjugal (com ou sem companheiro/a), filhos, escolaridade, tempo de profissão, tipo de função exercida e porte da empresa na qual trabalha.

\subsection{Procedimentos de coleta de dados}

A coleta de dados foi realizada por meio de instrumento on-line. Para o recrutamento dos participantes foi utilizada a técnica do Respondent Driven Sampling (RDS) que combina a amostragem em bola de neve (snowball sampling) (Goodman, 1961) e a utilização de redes sociais, como uma forma de compensar a amostragem não-aleatória (Heckathorn, 1997).

Num primeiro momento, foram identificadas as sementes da amostra, isto é, os sujeitos que pertenciam à população alvo do estudo ( $1^{\text {a }}$ onda) e, que poderiam recrutar novos participantes ( $2^{\mathrm{a}}$ onda), até que se tenha atingido o tamanho desejado da amostra (Goel \& Salganik, 2009). Essa é uma técnica que permite $o$ alcance de participantes com características semelhantes e que dispõem de tecnologia para acessar os instrumentos.

A amostra inicial foi contatada através da rede pessoal dos pesquisadores e ampliada através da técnica RDS. No convite constava o link para o preenchimento dos instrumentos no ambiente virtual Survey Monkey.

\subsection{Procedimentos de análise de dados}

Os dados referentes à descrição da amostra foram analisados mediante análise descritiva. O processo de adaptação do inventário envolveu os seguintes passos: tradução, retrotradução, pré-teste, estudo das evidências de validade de conteúdo e construto e análise de consistência interna. A 
análise das evidências de validade de construto foi realizada mediante análise fatorial confirmatória (AFC) com método de mínimos quadrados ponderados (WLSMV) e o uso do programa estatístico $R$, versão 3.3.0. As estatísticas descritivas dos itens do inventário e os alfas de Cronbach (consistência interna) foram realizadas utilizando-se o pacote estatístico SPSS, versão 21.

Quanto à análise fatorial confirmatória, para o modelo ser considerado satisfatório o índice de ajuste comparativo (Comparative Fit Index de Bentler, CFI) e o TLI (índice de Tucker e Lewis) devem ter valores próximos a 0,95 e o erro quadrático médio de aproximação (Root Mean Square Error of Approximation, RMSEA) menor que 0,08, incluindo seu intervalo de confiança (Byrne, 2010). Também são apresentadas as estatísticas descritivas dos itens e os alfas de Cronbach para cada uma das dimensões da escala.

\subsection{Aspectos éticos}

O estudo foi desenvolvido no período de março a setembro de 2014, após aprovação pelo Comitê de Ética em Pesquisa da Universidade Luterana do Brasil. Ao decidir participar da pesquisa on-line, os participantes eram direcionados a uma página com a explicação sobre os objetivos do estudo, as informações pertinentes à pesquisa e seus direitos, incluindo o anonimato da participação e o direito de desistir da participação da pesquisa a qualquer momento. Somente após manifestar conformidade com esse termo de consentimento para a participação é que os participantes eram direcionados à pagina relativa ao instrumento.

\section{RESULTADOS}

\subsection{Etapas do estudo de evidências de validade}

\subsubsection{Tradução e adaptação}

A adaptação brasileira do Inventário COPE seguiu os padrões metodológicos recomendados pela Comissão Internacional de Testes (ITC, 2016) para a adequação de instrumentos a outras culturas. Foram selecionados três tradutores qualificados com conhecimento do idioma inglês e português do Brasil: dois brasileiros que residiram na Inglaterra e um de nacionalidade americana e residente no Brasil. Foi realizado o processo de retrotradução (back-translation) e posterior comparação de versões. Na avaliação da equivalência semântica, verificou-se que o significado referencial apresentou bons resultados, tendo em vista que todos os itens obtiveram, nas retrotraduções, 
percentual de concordância acima de $80 \%$, valor considerado satisfatório, de acordo com Pasquali (2003).

A versão final em português foi submetida a um teste piloto com 30 trabaIhadores. Foi solicitado aos sujeitos que manifestassem aspectos relativos ao entendimento de palavras e enunciados. Nessa etapa, não houve necessidade de modificação de nenhum item. $O$ instrumento se mostrou de fácil compreensão, e o tempo médio de realização foi de 20 minutos.

\subsubsection{Análise de evidências de validade e fidedignidade}

\subsubsection{Evidências de validade de conteúdo}

Para acessar as evidências de validade de conteúdo e de construto do instrumento, esse foi encaminhado a cinco psicólogos, experts que atuam de acordo com o referencial da Psicologia Social e da Saúde. Esses deveriam manifestar-se por meio de um parecer acerca da compreensibilidade (escrita e clareza dos itens) do instrumento e sua relevância para a identificação das estratégias de enfrentamento. Os profissionais confirmaram a abrangência teórico-conceitual da escala, dando suporte à validade de conteúdo da versão em português do instrumento, e consideraram os itens apropriados para avaliar coping.

\subsubsection{Evidências de validade de construto}

Inicialmente, o inventário original com 60 itens foi testado por meio de análise fatorial confirmatória, analisando-se um modelo tridimensional (Foco no Problema, Foco na Emoção, Coping Evitativo). Todos os itens apresentaram pesos significativos na estimação do modelo. No entanto, conforme se observa na Tabela 1, os resultados apontam um modelo com índices de ajuste não aceitáveis (Modelo 1).

Dessa forma, optou-se por selecionar os dois itens com carga fatorial mais elevada de cada uma das 15 dimensões da COPE, totalizando 30 itens que foram agrupados em três dimensões: Foco no Problema (8 itens), Foco na Emoção (14 itens) e Coping Evitativo (8 itens) (Modelo 2). Os índices encontrados para o Modelo 2 foram melhorados, mas também não foram adequados (ver Tabela 1), assim optou-se por eliminar 12 itens que apresentaram pesos inferiores a 0,40 em sua respectiva dimensão. 0 modelo final da

escala (Modelo 3), composto por 18 itens (seis em cada dimensão) alcançou boa adequação, com índices de ajuste aceitáveis conforme se observa na Tabela 1 (Hooper, Coughlan, \& Mullen, 2008). 
Tabela 1.

Índices de ajuste das análises fatoriais confirmatórias da escala

\begin{tabular}{cccccccc}
\hline & $\mathbf{X}^{2}$ & $\mathbf{G L}$ & $\mathbf{P}$ & $\mathbf{X}^{2} / \mathbf{G L}$ & TLI & CFI & RMSEA (I.C. 95\%) \\
\hline Modelo 1-60 itens & 9158,80 & 1707 & $<0,001$ & 5,36 & 0,672 & 0,683 & $0,091(0,090-0,093)$ \\
Modelo 2-30 itens & 2008,38 & 402 & $<0,001$ & 4,99 & 0,764 & 0,782 & $0,087(0,084-0,091)$ \\
Modelo 3-18 itens & 348,16 & 133 & $<0,001$ & 2,61 & 0,933 & 0,942 & $0,056(0,049-0,063)$ \\
\hline
\end{tabular}

Na Figura 1, observa-se o modelo final da escala (Modelo 3) composto por 18 itens e três dimensões. Todos os parâmetros foram significativos, com pesos variando de 0,41 (item 35 do Coping Evitativo) a 0,71 (item 47 do Foco no Problema). Existem correlações significativas entre as dimensões Coping Evitativo e Foco na Emoção $(r=0,62, p<0,05)$ e entre o Coping Evitativo e Foco no Problema $(r=-0,25, p<0,05)$. No entanto, a correlação entre Foco no Problema e Foco na Emoção não alcançou significância estatística $(r=0,06$, $p=0,28)$, pelo que este parâmetro foi retirado do modelo.

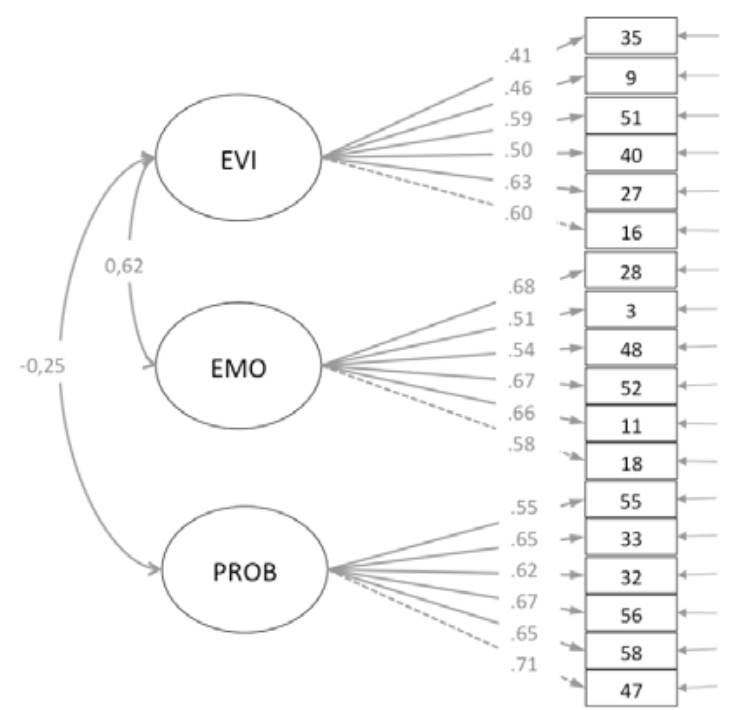

Figura 1. Modelo final (3) da escala COPE reduzida tridimensional. Pesos padronizados

\subsubsection{Estatísticas descritivas e consistência interna}

As estatísticas descritivas da COPE podem ser observadas na Tabela 2. As médias mais elevadas correspondem à dimensão Foco no Problema. A média mais elevada $(M=3,34)$ foi a do item 4 (Tento encontrar uma estratégia sobre o que fazer). A dimensão de Coping Evitativo apresentou as menores médias, sendo que o item 18 (Utilizo álcool ou medicação para pensar menos na situação) apresentou a menor média $(M=1,29)$. 
Em relação à correlação item-total corrigida, a maioria dos itens obteve valores superiores a $r=0,50$. Apenas os itens 11 , pertencente à subescala de foco na emoção e os itens 14, 15, 16, 17 e 18 da subescala de coping evitativo obtiveram correlações inferiores a 0,50. Quanto ao coeficiente de consistência interna, os valores foram acima de 0,70 para as três dimensões, e verifica-se que todos os itens contribuem para o alfa de Cronbach em seu fator de origem. Em relação à assimetria, todos os itens apresentaram um valor no intervalo de \pm 2 .

Tabela 2. Estatística descritiva e consistência interna dos itens e dimensões do Inventário COPE*

\begin{tabular}{|c|c|c|c|c|}
\hline SUBESCALA/ITEM & $M(D P)$ & $\begin{array}{c}\text { CORRELAÇÃO } \\
\text { ITEM-TOTAL } \\
\text { CORRIGIDA }\end{array}$ & $\begin{array}{l}\text { ASSIME- } \\
\text { TRIA }\end{array}$ & $\begin{array}{l}\text { ALFA } \\
\text { SEM ITEM }\end{array}$ \\
\hline Foco no Problema $(\alpha=0,81)$ & $3,01(0,60)$ & & 0,32 & \\
\hline 1 (47) Ação direta sobre o problema & $2,98(0,87)$ & 0,63 & $-0,58$ & 0,76 \\
\hline 2 (58) Faço passo a passo o que tem que ser feito & $3,16(0,80)$ & 0,55 & $-0,62$ & 0,78 \\
\hline 3 (56) Penso sobre que passos devo tomar & $3,19(0,79)$ & 0,58 & $-0,65$ & 0,77 \\
\hline 4 (32) Tento encontrar uma estratégia sobre o que fazer & $3,34(0,76)$ & 0,54 & $-0,82$ & 0,78 \\
\hline $\begin{array}{c}5 \text { (33) Concentro-me em lidar com o problema e afasto } \\
\text { outros temas da minha mente. }\end{array}$ & $2,93(0,87)$ & 0,58 & $-0,45$ & 077 \\
\hline $\begin{array}{c}6 \text { (55) Deixo de lado outras atividades para concentrar-me no } \\
\text { problema }\end{array}$ & $2,48(0,92)$ & 0,51 & $-0,03$ & 0,79 \\
\hline Foco na Emoção $(\alpha=0,78)$ & $2,31(0,69)$ & & 0,99 & \\
\hline 7 (11) Falo dos meus sentimentos com alguém & $2,44(0,98)$ & 0,55 & 0,20 & 0,73 \\
\hline 8 (52) Falo com alguém sobre como me sinto & $2,58(0,97)$ & 0,59 & $-0,04$ & 0,72 \\
\hline 9 (18) Peço a ajuda de Deus & $2,45(1,17)$ & 0,55 & 0,11 & 0,74 \\
\hline 10 (48) Tento encontrar conforto na religião & $2,17(1,09)$ & 0,53 & 0,50 & 0,74 \\
\hline 11 (3) Sinto-me angustiado e deixo aflorar minhas emoções & $2,10(0,88)$ & 0,38 & 0,35 & 0,77 \\
\hline 12 (28) Deixo aflorar meus sentimentos & $2,13(0,91)$ & 0,53 & 0,40 & 0,74 \\
\hline Coping Evitativo $(\alpha=0,71)$ & $1,66(0,54)$ & & 0,31 & \\
\hline $\begin{array}{c}13 \text { (27) Recuso-me a acreditar que a situação tenha } \\
\text { acontecido }\end{array}$ & $1,59(0,83)$ & 0,53 & 1,28 & 0,65 \\
\hline $\begin{array}{l}14 \text { (40) Ajo realmente como se o problema não tivesse } \\
\text { acontecido }\end{array}$ & $1,81(0,89)$ & 0,39 & 0,87 & 0,69 \\
\hline $\begin{array}{c}15 \text { (51) Reduzo a quantidade de esforço que dedico para } \\
\text { resolver o problema }\end{array}$ & $1,85(089)$ & 0,49 & 0,67 & 0,66 \\
\hline $\begin{array}{l}16 \text { (9) Admito que não posso lidar com a situação e deixo de } \\
\text { tentar }\end{array}$ & $1,40(0,69)$ & 0,43 & 1,78 & 0,68 \\
\hline $\begin{array}{l}17 \text { (16) Sonho acordado com outras coisas diferentes da } \\
\text { situação }\end{array}$ & $2,02(1,00)$ & 0,44 & 0,78 & 0,68 \\
\hline $\begin{array}{l}18 \text { (35) Utilizo álcool ou medicação para pensar menos na } \\
\text { situação }\end{array}$ & $1,29(0,74)$ & 0,40 & 1,63 & 0,69 \\
\hline
\end{tabular}

*Nota: Os números entre parênteses para cada item correspondem aos números apresentados na Figura1. 


\section{DISCUSSÃO}

O presente estudo objetivou avaliar a estrutura fatorial do Inventário COPE em uma amostra de trabalhadores brasileiros, mediante AFC. Os resultados corroboraram o modelo hipotetizado de três fatores (Endler \& Parker, 1990), apresentando resultados adequados com evidências de validade e confiabilidade para seu uso no contexto brasileiro.

A opção pela redução de itens do Inventário COPE completo ao invés da adoção do Brief COPE deve ser considerada. Enquanto o Brief COPE, com 28 itens, abarca 14 estratégias de enfrentamento do estresse (Carver, 1997), a versão obtida, com 18 itens, contempla as três dimensões gerais do modelo de coping (Endler \& Parker, 1990), avaliadas até mesmo em estudos com outros instrumentos (Khorasani \& Ghanizadeh, 2017). A Brief COPE permite uma análise mais pormenorizada das estratégias, ainda que cada estratégia seja avaliada com apenas dois itens. A versão obtida no presente estudo apresenta 10 itens a menos que a Brief COPE e permite avaliar as três dimensões mais globais de coping, o que também lhe confere maior robustez. Em relação à versão francesa da Brief COPE, de quatro dimensões (Baumstarck et al., 2017), a versão apresentada diferencia-se por ter sido obtida mediante uma amostra sem um estressor vital específico.

Quanto aos resultados obtidos na análise de dados, os valores da correlação item-total corrigida foram elevados, todos superiores a 0,38, evidenciando que todos os itens contribuem para sua função combinada (Streiner \& Norman, 1995). Cada dimensão do COPE pode ser considerada com uma função linear dos itens que a compõem. Todos os itens atendem ao pressuposto de normalidade univariada, com valores dos índices de assimetria inferiores a dois (Curran, West, \& Finch, 1996). Os valores de alfa de Cronbach obtidos em cada dimensão, aliados à coerência semântica do conjunto de itens, permitem considerar que todos contribuem para o estabelecimento do construto ao qual pertencem originalmente. Quanto à consistência interna das dimensões, os valores obtidos mostram-se satisfatórios (Tabachnick \& Fidell, 2014).

As correlações encontradas entre os fatores Foco na Emoção e Coping Evitativo foi de $r=0,62(p<0,001)$, entre o Foco no Problema e Coping Evitativo de $r=$ $-0,25(p<0,001)$, e entre Foco no Problema e Foco na Emoção não foi significativa. Todos os itens apresentaram carga fatorial significativa. $O$ menor valor obtido foi pelos itens $18(\lambda=0,41)$ e $16(\lambda=0,46)$. 
O presente estudo teve por objetivo adaptar e reduzir a escala, a partir de sua estrutura original de 60 itens. Nesse sentido, ressalta-se que os diversos aspectos de evidências de validade do instrumento reduzido apresentaram resultados satisfatórios, indicando que o COPE possui propriedades psicométricas adequadas para o estudo do coping no Brasil.

Como limitação do estudo é importante destacar que a maioria da amostra é composta de trabalhadores de empresas de médio e grande porte. É, portanto, importante, ampliar a amostra para os demais segmentos, como o de serviços, rural, entre outros, a fim de verificar se a escala mantém as mesmas propriedades psicométricas nos diversos grupos de trabalhadores.

A partir dos resultados encontrados e das limitações do presente estudo, também se sugere que sejam conduzidas novas investigações de validade do Inventário COPE. De acordo com a proposta do estudo de validação do COPE, é interessante que futuros estudos possam avaliar as relações da escala com outras medidas relacionadas ao coping, buscando avaliar evidências de validade convergente e divergente, como escalas de bem-estar e qualidade de vida ou escalas de estresse e depressão.

Financiación: Síndrome de Burnout e estratégias de enfrentamento entre trabalhadores. Universidade Federal de Ciências da Saúde de Porto Alegre (UFCSPA). Grupo de Pesquisas em Psicologia e Processos de Saúde (GPS). Financiamento próprio.

Agradecimientos: Agradecemos aos trabalhadores que disponibilizaram seu tempo para contribuir para este estudo.

\section{REFERÊNCIAS}

Antoniazzi, A. S. (2000). Desenvolvimento de instrumentos para a avaliação de coping em adolescentes brasileiros. (Tese de Doutorado). Universidade Federal do Rio Grande do Sul. Porto Alegre, Brasil. http://hdl.handle.net/10183/2908

Baumstarck, K., Alessandrini, M., Hamidou, Z., Auquier, P., Leroy, T., \& Boyer, L. (2017). Assessment of coping: A new french four factor structure of the Brief Cope Inventory. Health and Quality of Life Outcomes, 15(8), 1-9. http://dx.doi. org/10.1186/s12955-016-0581-9

Biggs, A., Brough, P., \& Drummond, S. (2017). Lazarus and Folkman's Psychological Stress and Coping Theory. In C. L. Cooper \& J. Campbell (Eds.), The Handbook of Stress and Health: A Guide to Research and Practice (pp.351-364). West Sussex: John Wiley \& Sons.

Byrne, B. M. (2010). Structural equation modeling with AMOS. Basic concepts, applications and programming $\left(2^{\text {nd }} e d.\right)$. New York: Routledge. 
Câmara, S., \& Carlotto, M. (2016). Escala Brasileira de Coping para Adolescentes (EBCA): Versão revisada. Psicologia em Pesquisa, 10(1), 94-102. http://dx.doi. org/10.24879/201600100010051

Cabral, J., \& Matos, P. M. (2010). Cope-Inventory: teste da estrutura factorial com uma amostra de jovens adultos universitários. Psicologia, XXIV(1), 49-71. http://www. scielo.mec.pt/scielo.php?script=sci_arttext\&pid=S0874-20492010000100003

Carver, C. S. (1997). You want to measure coping but your protocol's too long: Consider the Brief COPE. International Journal of Behavioral Medicine, 4, 92-100. Doi: http://dx.doi.org/10.1207/s15327558ijbm0401_6

Carver, C. S., \& Scheier, M. F. (1981). Attention and self-regulation: A control-theory approach to human behavior. New York: Springer- Verlag.

Carver, C. S., \& Scheier, M. F. (2017). Optimism, coping, and well-being. In C. L. Cooper \& J. Campbell (Eds.), The Handbook of Stress and Health: A Guide to Research and Practice (pp. 400-414). West Sussex: John Wiley \& Sons.

Carver, C. S., Scheier, M. F., \& Weintraub, B. (1989). Assessing coping strategies: A theoretically based approach. Journal of Personality and Social Psychology, 56(2), 267-283. http://dx.doi.org/10.1037/0022-3514.56.2.267

Carver, C. S. \& Connor-Smith, J. (2010). Personality and coping. Annual Review of Psychology, 61(1), 679-704. https://doi.org/10.1146/annurev. psych.093008.100352.

Collage of arts and sciences (2018). Profesor Charles S. Caver. Recuperado en http:// www.psy.miami.edu/faculty/charles-s-carver-phd/

Collage of arts and sciences (2018). Página principal. Recuperado en http://www.psy. miami.edu/faculty/charles-s-carver-phd/

Curran, P. J., West, S. G., \& Finch, J. F. (1996). The robustness of test statistics to nonnormality and specification error in confirmatory factor analysis. Psychological. Methods, 1(1), 16-29. Doi: https://doi.org/10.1037//1082-989x.1.1.16.

Diniz, S., \& Zanini, D. (2010). Relação entre fatores de personalidade e estratégias de coping em adolescentes. Psico-USF, 15(1), 71-80. http://dx.doi.org/10.1590/ s1413-82712010000100008

Dobreva-Martinova, T., Villenueve, M., Strickland, L., \& Matheson, K. (2002). Occupational role stress in the Canadian forces: Its association with individual and organizational well-being. Canadian Journal of Behavioural Science, 34(2), 111-121. https://doi.org/10.1037/h0087161

Endler, N. S., \& Parker, J. D. (1990). Multidimensional assessment of coping: A critical evaluation. Journal of Personality and Social Psychology, 58(5), 844-854. https:// doi.org/10.1037/0022-3514.58.5.844

Folkman, S., \& Lazarus, R. S. (1980). An analysis of coping in a middle-aged community sample. Journal of Health and Social Behavior, 21(3), 219-239. https://doi. org/10.2307/2136617

Folkman, S., \& Lazarus, R. S. (1985). If it changes, it must be a process: A study of emotion and coping during three stages of a college examination. Journal of Personality and Social Psychology, 48, 150-170. http://dx.doi.org/10.1037/00223514.48.1.150

Frydenberg, E. (2017). The Measurement of Coping. In E. Frydenberg, Coping and the Challenge of Resilience (pp.47-66). London: Palgrave Macmillan. https://doi. org/10.1057/978-1-137-56924-0_4

Goel, S., \& Salganik, M. J. (2009). Respondent-driven sampling as Markov chain Monte Carlo. Statistics in Medicine, 28(17), 2202-2229. https://doi.org/10.1002/ sim.361 
Goodman, L. A. (1961). Snowball sampling. Annuals Mathematic Statistics, 32(1), 148-170. https://doi.org/10.1214/aoms/1177705148

Greer, T. (2007). Measuring Coping Strategies Among African Americans: An Exploration of the Latent Structure of the COPE Inventory. Journal of Black Psychology, 33(3), 260-277. http://dx.doi.org/10.1177/0095798407302539

Heckathorn, D. D. (1997). Respondent driven sampling: A new approach to the study of hidden populations. Social Problems, 44(2), 174-199. https://doi.org/10.1525/ sp.1997.44.2.03×0221m

Hooper, D., Coughlan, J., \& Mullen, M. R. (2008). Structural equation modeling: Guidelines for determining model fit. Electronic Journal of Business Research Methods, 6(1), 53-60. Recuperado de https://arrow.dit.ie/cgi/viewcontent. cgi ?article $=1001 \&$ context=buschmanart

International Test Commission (2016). The ITC guidelines for translating and adapting tests (Second edition). Recuperado de www.InTestCom.org

Juth, V., Dickerson, S. S., Zoccola, P. M., \& Lam, S. (2015). Understanding the utility of emotional approach coping: evidence from a laboratory stressor and daily life. Anxiety, Stress, and Coping, 28(1), 50-70. http://doi.org/10.1080/10615806.201 4.921912

Kallasmaa, T., \& Pulver, A. (2000). The structure and properties of the Estonian COPE inventory. Personality and Individual Differences, 29(5), 881-894. http://dx.doi. org/10.1016/s0191-8869(99)00240-8

Kato, T. (2013). Frequently used coping scales: A meta-analysis. Stress \& Health, 31(4), 315-323. https://doi.org/10.1002/smi.2557

Khorasani, R., \& Ghanizadeh, A. (2017). The role of coping strategies and emotional factors in predicting Iranian translation students' translation ability. Asia Pacific Translation and Intercultural Studies, 1-15. http://dx.doi.org/10.1080/23306343 .2017 .1346461

Latack, C. J. (1986). Coping with job stress: measures and future directions for scale development. Journal of Applied Psychology, 71(3), 377-385. https://doi. org/10.1037/0021-9010.71.3.377

Lazarus, R. S. (2006). Emotions and interpersonal relationships: toward a person-centered conceptualization of emotions and coping. Journal of Personality, 74(1), 9-46. https://doi.org/10.1111/j.1467-6494.2005.00368.x

Lazarus, R. S., \& Folkman S. (1984). Estresse, appraisal, and coping. New York: Springer.

Lyne, K., \& Roger, D. (2000). A psychometric re-assessment of the COPE questionnaire. Personality and Individual Differences, 29(2), 321-335. https://doi.org/10.1016/ s0191-8869(99)00196-8

Litman, J. A. (2006). The COPE inventory: dimensionality and relationships with approach - and avoidance motives and positive and negative traits. Personality and Individual Differences, 41(2), 273-284. https://doi.org/10.1016/j. paid.2005.11.032

Marroquín B., Tennen H., \& Stanton A. L. (2017). Coping, emotion regulation, and well-being: Intrapersonal and Interpersonal Processes. In M. Robinson \& M. Eid, (eds), The happy mind: cognitive contributions to well-being (pp. 253-274). Cham: Springer. https://doi.org/10.1007/978-3-319-58763-914_

Melo, L. P., Carlotto, M. S., Rodriguez, S. Y. S., \& Diehl, L. (2016). Estratégias de enfrentamento (coping) em trabalhadores: revisão sistemática da literatura nacional. Arquivos Brasileiros de Psicologia, 68(3), 125-144. http://pepsic.bvsalud.org/ scielo.php?pid=S1809-52672016000300010\&script=sci_abstract\&tIng=es 
Morero, J. A. P., Bragagnollo, G. R., \& Santos, M. T. S. (2018). Estratégias de enfrentamento: uma revisão sistemática sobre instrumentos de avaliação no contexto brasileiro. Revista Cuidarte, 9(2), 2257-2268. https://dx.doi.org/10.15649/ cuidarte.v9i2.503

Murta, S., \& Tróccoli, B. (2007). Stress ocupacional em bombeiros: Efeitos de intervenção baseada em avaliação de necessidades. Estudos de Psicologia (Campinas), 24(1), 41-51. http://dx.doi.org/10.1590/s0103-166x2007000100005

Noda, T., Takahashi, Y. \&, Murai, T. (2018). Coping mediates the association between empathy and psychological distress among Japanese workers. Personality and Individual Differences, 124, 178-183. https://doi.org/10.1016/j.paid.2017.12.009

O'Driscoll, M. P. (2013). Coping with stress: A challenge for theory, research and practice. Stress and Health, 29(2), 89-90. https://doi.org/10.1002/smi.2489

Pasquali, L. (2003). Psicometria: teoria dos testes na Psicologia e na Educação. Petrópolis: Vozes.

Pinheiro, F. A., Tróccoli, B. T., \& Tamayo, M. R. (2003). Mensuração de coping no ambiente ocupacional. Psicologia: Teoria e Pesquisa, 19(2), 153-158. https:// dx.doi.org/10.1590/S0102-37722003000200007

Reeck, C., Ames, D. R., \& Ochsner, K. N. (2016). The social regulation of emotion: An integrative, cross-disciplinary model. Trends in Cognitive Sciences, 20, 47-63. http://dx.doi.org/10.1016/j.tics.2015.09.003_

Roche, A., \& Ogden, J. (2017). Predictors of burnout and health status in Samaritans' listening volunteers. Psychology, Health \& Medicine, 22(10), 1169-1174. http:// dx.doi.org/10.1080/13548506.2017.1280176

Seidl, E. M. F., Troccoli, B. T., \& Zannon, C. M. L. C. (2001). Análise fatorial de uma medida de estratégia de enfrentamento. Psicologia: Teoria e Pesquisa, 17(3), 225-34. https://doi.org/10.1590/s0102-37722001000300004

Schaufeli, W., \& Enzmann, D. (1998). The burnout companion to study and practice A critical analysis. London: Taylor \& Francis.

Sinha, R., Lacadie, C., Constable, R., \& Seo, D. (2016). Dynamic neural activity during stress signals resilient coping. Proceedings of The National Academy of Sciences, 113(31), 8837-8842. http://dx.doi.org/10.1073/pnas.1600965113

Streiner, D. L., \& Norman, G. R. (1995). Health measurement scales: A practical guide to their development and use ( $2^{\text {nd }}$ ed.). New York: Oxford University Press.

Tabachnick, B. G., \& Fidell, L. S. (2014). Using Multivariate Statistics. (6 ed.). Boston: Pearson Education.

Veloso-Besio, C., Caqueo-Arancibia, W., Caqueo-Urízar, A., Muñoz-Sánchez, Z., \& Villegas-Abarzúa, F. (2010). Estrategias de afrontamiento en adolescentes. Fractal: Revista de Psicologia, 22(1), 23-34. http://dx.doi.org/10.1590/s198402922010000100003 autor o el licenciante.

\section{(cc) BY}

